\title{
A Case of Congenital Tuberculosis Born from a Mother with Pulmonary Tuberculosis
}

Hoan Jong Lee ${ }^{1,2}$, Hee-Sup Kim ${ }^{3}$, Eun Hwa Choi ${ }^{1,2}$

1Department of Pediatrics, Seoul National University Children's Hospital

2Department of Pediatrics, Seoul National University College of Medicine, Seoul, Korea

${ }^{3}$ Department of Pediatrics, Dongguk University Ilsan Hospital, Goyang, Korea

\section{Background}

- Congenital tuberculosis is a rare disease with a high fatality rate. Congenital tuberculosis is thought to be infected through the placenta form the infected mother. We report a case of congenital tuberculosis in a 15-day-old male infant who presented with fever and vomiting.

\section{Case summary}

\section{- Brief History}

A fifteen-day-old male infant was admitted with fever and intermittent vomiting for 3 days. The baby was born by vaginal delivery from a mother who had a history of salpingectomy due to unknown cause. The baby was transferred from outside hospital due to persistent fever despite the administration of ampicillin and cefotaxime.

\section{- Physical Examination}

The baby remains febrile. Distended abdomen was noted with engorged superficial vein and

hepatosplenomegaly.

\section{- Work-up for Tuberculosis}

Tuberculin skin test found to be negative. Interferon-gamma assay was positive. Gene Expert PCR of gastric aspirates were positive while the smear was negative.

\section{- Diagnostic Images}

Abdominal sonography revealed multiple microabscesses on liver, spleen and kidney.

There were no pulmonary lesions on chest CT. Nodular enhancing lesions in the bilateral cerebral and cerebellar hemisphere on brain MRI suggested tuberculous granuloma (Figure 1).

The baby's mother had no apparent clinical symptoms but her chest radiograph showed micronodular infiltrates on the left upper lobe. Sputum specimen revealed acid-fast bacilli, and M. tuberculosis was cultured from sputum (Figure 1).

\section{- Clinical Course}

Smears of intraabdominal LN specimens contained acid-fast bacilli, and cultures were positive for Mycobacterium tuberculosis. (Figure 2)

The baby was managed with isoniazid, pyrazinamide, rifampicin for 12 months. During the course of management, the baby developed massive ascites because of occlusion of main portal vein Ascites drainage was performed due to respiratory distress (Figure 3 ).

\section{Conclusion}

Congenital tuberculosis may be considered in infants with fever and intraabdominal microabscess which do not respond to antibiotic treatment
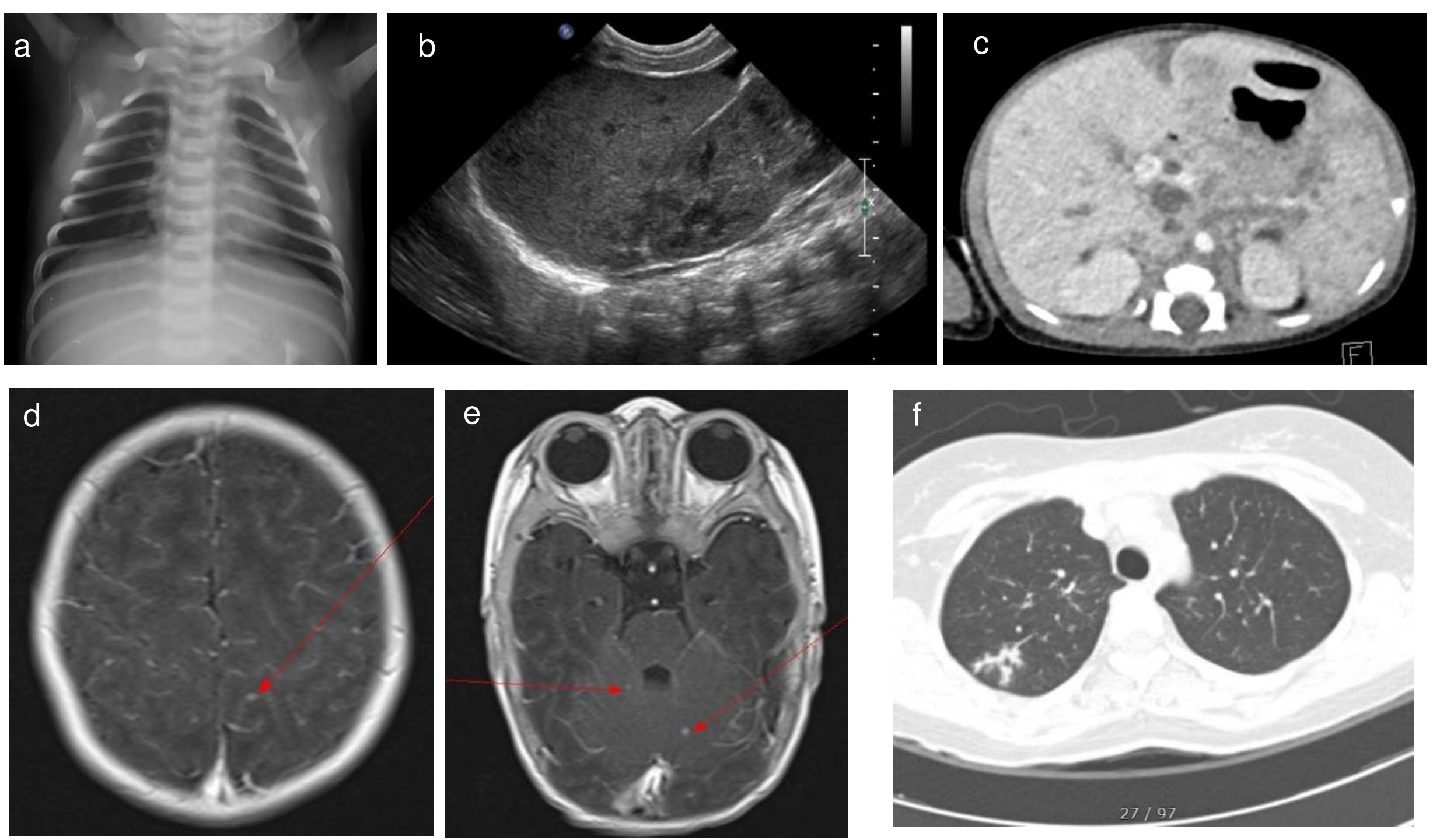

Figure 1. Diagnostic Images: a) Normal chest x-ray, b, c) Abdominal sonography and CT revealing multiple microabscesses on liver, spleen and kidney, d, e) Nodular enhancing lesions in the bilateral cerebral and cerebellar hemisphere on brain MRI, f) Mother's Chest CT: micronodular infiltrates on the left upper lobe suggesting active pulmonary tuberculosis

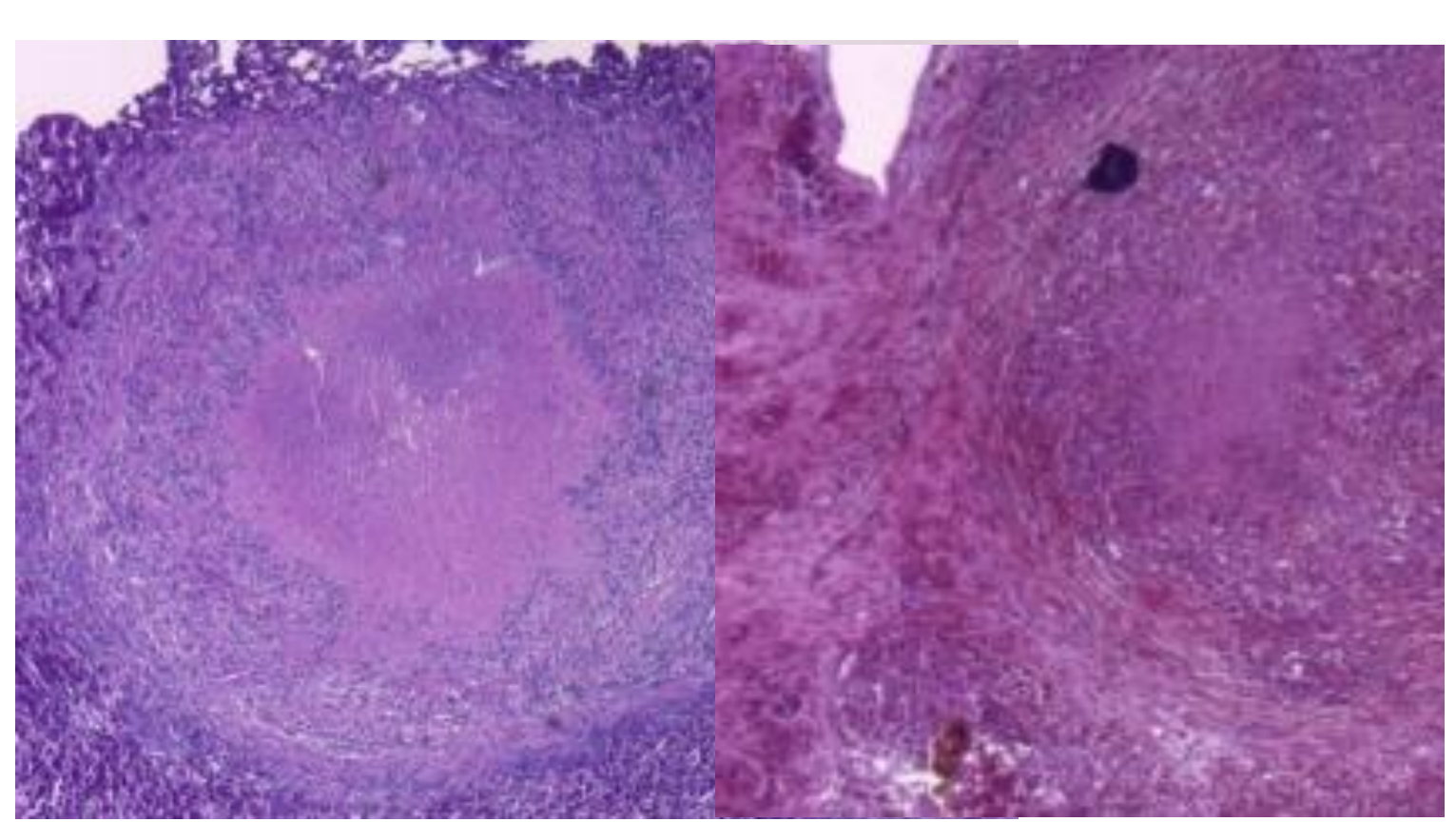

Figure 2. Intraabdominal Lymph node revealing chronic granulomatous inflammation with caseous necrosis and confluent granuloma. AFB staining reveals acid-fast bacilli

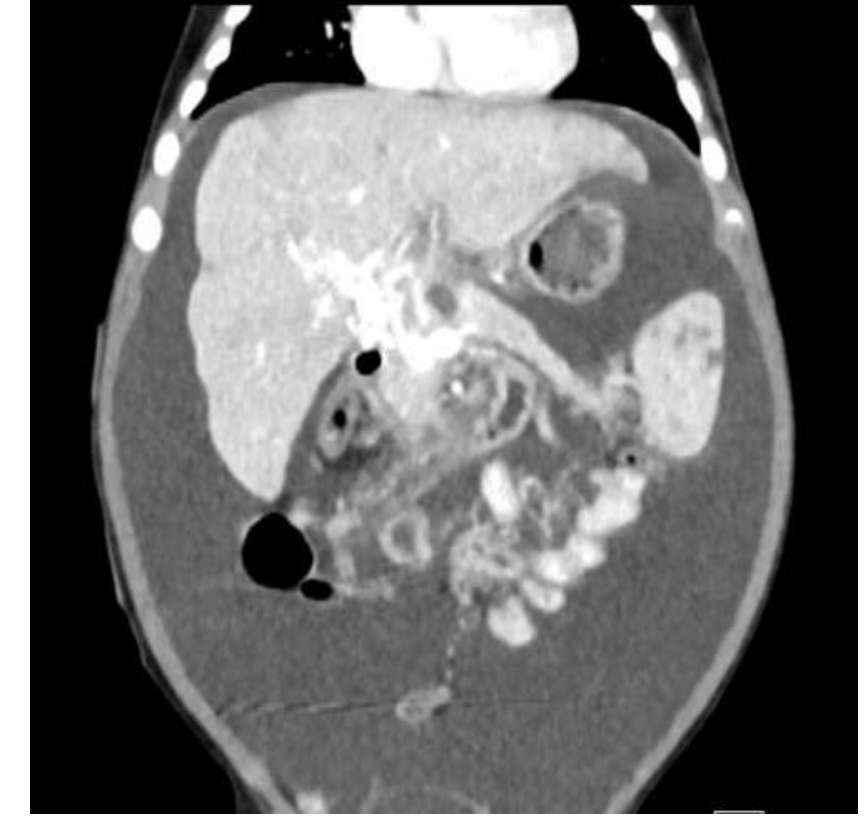

Figure 3. Occlusion of main portal vein with cavernous transformation with massive ascites. 